\title{
PAG Study in EUV Lithography
}

\author{
Toshikage Asakura, Hitoshi Yamato, Yuichi Nishimae, \\ Keizou Okada and Masaki Ohwa \\ Microelectronics, Emerging Competencies Amagasaki \\ Ciba Japan K.K., 7-1-13, Doi-cho, Amagasaki, Japan 660-0083
}

Extreme Ultraviolet (EUV) has already achieved the initial requirements for $32 \mathrm{~nm}$ DRAM half pitch lithography rule and is known as one of the most promising next generation lithography techniques to be realized for $22 \mathrm{~nm}$ patterning technology though strict requirements for the power of the light sources, lithographic performance of the photoresist and the manufacturing and inspection of masks are still remaining,.

In this report, we investigated the photolithographic characteristics of Photoacid Generator (PAG) additive approach in EUV lithography with different polymer platforms and sulfonium-type PAGs compared with other exposure techniques to understand the relationship between lithography results and photoresist materials. Four different sulfonium nonafluorobutanesulfonate; triphenylsulfonium nonafluorobutanesulfonate (TPS), tri(4methoxy-3,5-dimethylphenyl) sulfonium nonafluorobutanesulfonate (MDP), tri(4-methoxy-3methylphenyl)sulfonium nonafluorobutanesulfonate (MMP) and tri(4-methoxy-3phenylphenyl)sulfonium nona-fluorobutanesulfonate (MPP) were employed as PAG in order to study the fundamental properties, such as sensitivity, photo-efficiency, lithographic performance, in the two different model formulations, poly(hydroxystyrene) (PHS) type and poly(methacrylates)-type, under three different exposures of $193 \mathrm{~nm}$ (ArF), Electron Beam (EB) and EUV.

Keywords: ionic, photoacid generator, EUV, chemically amplified resist<smiles>c1ccc([S+](c2ccccc2)c2ccccc2)cc1</smiles>

TPS<smiles>COc1c(C)cc([S+](c2cc(C)c(OC)c(C)c2)c2cc(C)c(OC)c(C)c2)cc1C</smiles>

MDP<smiles>COc1ccc([S+](c2ccc(OC)c(-c3ccccc3)c2)c2ccc(OC)c(-c3ccccc3)c2)cc1-c1ccccc1</smiles>

MPP<smiles>COc1ccc([S+](c2ccc(OC)c(C)c2)c2ccc(OC)c(C)c2)cc1C</smiles>

MMP

\section{PAG employed in this study}

(nf: anion of nonafluorobutanesulfonic acid )

\section{Introduction}

Further miniaturization has always been a duty in semiconductor fabrication and technology developments related to photoresist lithography have been achieved with a drastic speed to meet the demand. The latest technology, which is currently $193 \mathrm{~nm}$ (ArF) photoresist lithography, has achieved the semiconductor fabrication with a design rule at $50 \mathrm{~nm}$ DRAM half pitch (HP) scaling technology. Though ArF lithography is expected to be extended to the $32 \mathrm{~nm}$ scaling rule owing remarkable progress in new technology like double-patterning, the limitation of utilization is also expected due to 
its high cost and low yield for the complicated manufacturing processes.

Extreme Ultraviolet (EUV) has already achieved the initial requirements for $32 \mathrm{~nm}$ DRAM HP lithography rule and is known as one of the most promising next generation lithography techniques to realize $22 \mathrm{~nm}$ scaling technology though strict requirements for the power of the light sources, lithographic performance of the photoresist and the manufacturing and inspection of masks are still remaining. [1]

Regarding the lithographic performance on EUV exposure, the well-known three serious challenges for photoresists are sensitivity, resolution and linewidth roughness (LWR); target criteria are below $10 \mathrm{~mJ} / \mathrm{cm}^{2}$ in sensitivity, below $40 \mathrm{~nm}$ in HP resolution, and below $3 \mathrm{~nm} \mathrm{(3 \sigma )}$ in LWR simultaneously. [2] To achieve the criteria, many approaches and concepts, such as molecular glass and PAG on polymers, are investigated and developed thus far. [3-4]

In this report, we investigated the fundamental properties of authentic PAG additive approach in EUV lithography with different polymer platforms and different sulfonium-type PAGs compared with other exposure techniques to understand the relationship between lithography results and photoresist materials.

We utilized EUV Micro-Exposure Tool (eMET) in Sematech North at Albany in the US for $50 \mathrm{~nm}$ line and space (L/S) pattering lithography comparing EUV photoresist model formulations in order to study the basic properties, such as sensitivity, photo-efficiency, exposure latitude and line width roughness. [5] Four different sulfonium nonafluorobutanesulfonate; triphenylsulfonium nonafluorobutanesulfonate (TPS), tri(4-methoxy3,5-dimethylphenyl) sulfonium nonafluorobutanesulfonate (MDP), tri(4-methoxy-3methylphenyl)sulfonium nona-fluorobutanesulfonate (MMP) and tri(4-methoxy-3phenylphenyl)sulfonium nona-fluorobutanesulfonate (MPP) were employed in the two different model formulations, poly(hydroxystyrene) (PHS) type and poly(methacrylates)-type, under three different exposures of ArF, Electron Beam (EB) and EUV.

Table 1. Composition of ArF formulations

\begin{tabular}{|c|c|}
\hline Components & Parts (Weight) \\
\hline Binder polymer & 100 \\
\hline PAG & 2 \\
\hline PGMEA & 1300 \\
\hline
\end{tabular}

\section{Experimental}

2.1. Photolithographic properties in $\mathrm{ArF}$ model formulation

To evaluate the photographic properties of our model formulation, we irradiated them by open frame exposure with VUVES 4500 mini, Litho Tech Japan, as an ArF exposure tool. Our positive tone resist model formulations utilized a copolymer of $\gamma$-butyrolactone methacrylate and 2-methyladamantyl methacrylate (62/38 mol-\%, Mitsubishi Rayon Co., Ltd) having a weight average molecular weight of 11800 . The composition of the formulation is described in Table 1.

The resist was spin-coated at $120 \mathrm{~nm}$ thickness on silicon wafers on which the bottom antireflection coating (ARC-29, Nissan Chemical) was applied at $82 \mathrm{~nm}$ thickness in advance and prebaked at $200^{\circ} \mathrm{C}$ for $60 \mathrm{sec}$. After exposure with various doses of energy, a post exposure bake was applied at $120^{\circ} \mathrm{C}$ for $60 \mathrm{sec}$ and the photoresist was then developed in $2.38 \%$ aqueous tetramethylammonium hydroxide solution, NMD-3 commercially available from $\mathrm{TOK}$, for $60 \mathrm{sec}$ at $23^{\circ} \mathrm{C}$.

\subsection{Photolithographic properties in EB model formulation}

To evaluate the EB sensitivity of our model formulation, the EB model photoresist formulations were irradiated by open frame radiation with JBX500SI, JEOL, as an exposure tool at Technology Research Institute of Osaka Prefecture. The acceleration voltage for patterning was $50 \mathrm{keV}$. Our positive tone photoresist model formulations utilized two copolymers, one is PHS-type (Copolymer A) and the other is poly(methacrylates)-type (Copolymer B). The composition of formulations is described in Table 2. As for PAG loading, the same molar amount of PAG is loaded as 4 parts of TPS.

Table 2. Composition of EB/EUV formulations

\begin{tabular}{|c|c|}
\hline Components & Parts \\
\hline Binder polymer & 100 \\
\hline PAG & $4 \sim 5.2$ \\
\hline N-1-adamantylacetamine & 0.27 \\
\hline $\begin{array}{c}\text { PGMEA/Ethyl lactate } \\
\text { (7/3 by volume) }\end{array}$ & 3000 \\
\hline
\end{tabular}

The photoresist was spin-coated at $60 \mathrm{~nm}$ thickness on silicon wafers on which surface 1,1,1,3,3,3hexamethyldisilazane commercially available Tokyo Chemical Industry was treated at $40^{\circ} \mathrm{C}$ and 
soft-baked at $110^{\circ} \mathrm{C}$ for $60 \mathrm{sec}$. After radiation process with various doses of energy, a post exposure bake was applied at $110^{\circ} \mathrm{C}$ for $60 \mathrm{sec}$ and the photoresist was then developed at $23^{\circ} \mathrm{C}$ in NMD-3 for $60 \mathrm{sec}$.

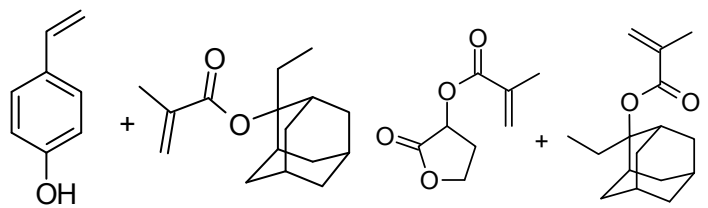

Copolymer A Copolymer B

2.3. Photolithographic properties in EUV model formulation

To evaluate the EUV photolithographic properties, the $50 \mathrm{~nm} \mathrm{~L} / \mathrm{S}$ patterning was performed with the EUV model photoresist formulations by eMET in Sematech North at Albany in the US, as exposure equipment. The composition of the formulation is the same as described in Table 2.

In the lithography process, the photoresistcoating and baking processes are the same as the ones applied in EB evaluation processes. Critical dimension of the obtained line was measured with CD SEM at Sematech North and LWR was determined as a indicator for roughness estimation by software analysis with SuMMIT purchased from EUV Technology, CA, in the US.

2.4. UV absorption and transparency measurements PAGs' UV absorption spectra in acetonitrile with $1 \mathrm{~cm}$ optical path-length were taken with a Hitachi U-3300 spectrometer. Absorbance at $193 \mathrm{~nm}$ was quantified in a $0.01 \mathrm{~g} / \mathrm{L}$ solution in acetonitrile.

As for the PAG absorption at $13.5 \mathrm{~nm}$, we estimated by the calculation method developed by the Center for X-Ray Optics, Lawrence Berkeley National Laboratory in the US. [6]

\section{Results and discussion}

3.1 Photolithographic properties in ArF model formulation

ArF lithography is the most advanced photolithography technique for high volume production currently. In order to investigate the photolithographic properties of $\mathrm{PAG}$ for $\mathrm{ArF}$ photoresist applications, each PAG was formulated in an $\mathrm{ArF}$ model formulation with $2 \mathrm{wt} \%$ against polymer, respectively. As the polymer matrix, a typical poly(methacrylates) platform was employed which has high transparency at $193 \mathrm{~nm}$. The photoresist layer was exposed by ArF laser (193 $\mathrm{nm})$ to determine the sensitivity. In lithography studies, the "Dose to clear" $\left(\mathrm{E}_{0}\right)$ is employed as a measure of photoresist sensitivity. The $\mathrm{E}_{0}$ is the dose of energy just sufficient to remove the photoresist film at the development process completely. As the required dose decreases, the sensitivity of the photoresist formulation increases.

Table 3. Sensitivity $\left(\mathrm{E}_{0}\right)$, Absorbance at $193 \mathrm{~nm}$ and Pparameter

\begin{tabular}{|c|c|c|c|}
\hline PAG & $\begin{array}{c}\mathbf{E}_{\mathbf{0}} \\
\left(\mathbf{m J} / \mathbf{c m}^{\mathbf{2}}\right)\end{array}$ & $\begin{array}{c}\text { Abs. } \mathbf{a} \\
\mathbf{1 9 3} \mathbf{~ n m}\end{array}$ & P-parameter \\
\hline TPS & 1.6 & 1.26 & 2.0 \\
\hline MDP & 4.9 & 0.78 & 3.9 \\
\hline MMP & 5.4 & 0.72 & 3.9 \\
\hline MPP & 9.9 & 0.82 & 8.1 \\
\hline
\end{tabular}

The obtained $\mathrm{E}_{0}$ for each PAG is listed in the Table 3 with the PAG absorbance at $193 \mathrm{~nm}$, which was obtained in acetonitrile solution at $0.01 \mathrm{~g} / \mathrm{L}$ concentration with $1 \mathrm{~cm}$ optical path-length, and Pparameter. [7] To evaluate PAG photo-efficiency in photoresist formulations, P-parameter, which is defined by the value of sensitivity $\left(\mathrm{E}_{0}\right)$ multiplied by PAG absorbance listed in Table 3, was employed here. It is notable that the differences in absorption and p-parameter are only due to PAG's chromophore difference in performance because the same acid, nonafluorobutanesulfonic acid, was generated from each PAG in this study. The smaller p-parameter value indicates the higher photoefficiency. The P-parameter normalized by the TPS value is plotted in Figure 1. TPS exhibited the highest photo-efficiency among the PAGs evaluated here. MDP and MMP followed TPS and were superior to MPP in terms of photo-efficiency.

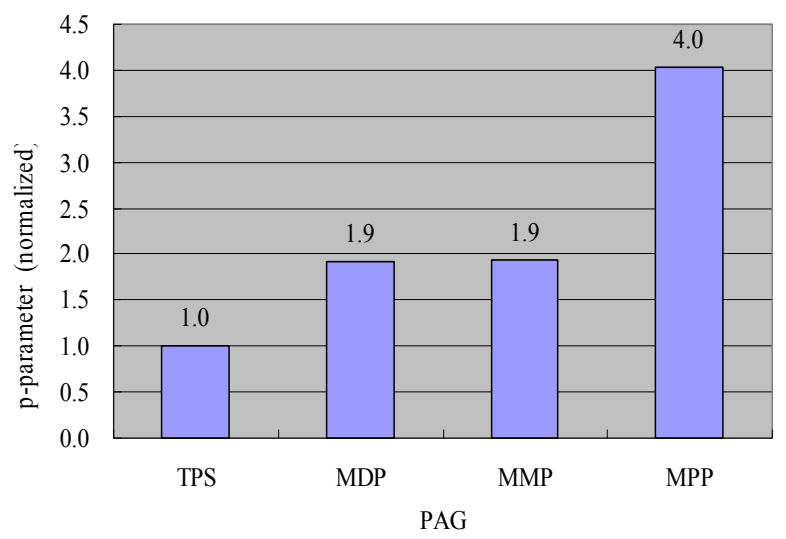

Figure 1. P-parameter (normalized) comparison

\subsection{Photolithographic properties in EB model} formulation

EB radiation chemistry is often studied to interpret the results with EUV lithography results. We 
evaluated the photo-sensitivity, $\mathrm{E}_{0}$, with the $\mathrm{EB}$ exposure tool. Here one of the important differences between EB radiation lithography and EUV exposure one is energy of doses. While EUV exposure, which is $13.5 \mathrm{~nm}$ wavelength, corresponds to $92.5 \mathrm{eV}$ of energy, a typical acceleration voltage in EB lithography, $50 \mathrm{keV}$, is used here.

The sensitivity results, $E_{0}$, in two formulations with different polymer matrixes, Copolymer A including PHS and Copolymer B comprising poly(methacrylates), are plotted in Figure 2. The formulations with Copolymer A showed higher photospeed than the ones with Copolymer B in the presence of any PAGs. Especially in case of MDP, $\mathrm{E}_{0}$ with Copolymer A was less than half of that with Copolymer B. Kozawa et al. reported lower acid generation efficiency in poly(methyl methacrylate) (PMMA) than PHS under EB radiation generally because secondary electron generated from polymer matrix is trapped by PMMA itself and because the deprotonation efficiency of PMMA radical cations is lower than that of PHS radical cations. [8] The phenomena observed in PMMA may be one of the reasons why such a drastic sensitivity difference between the two was observed.

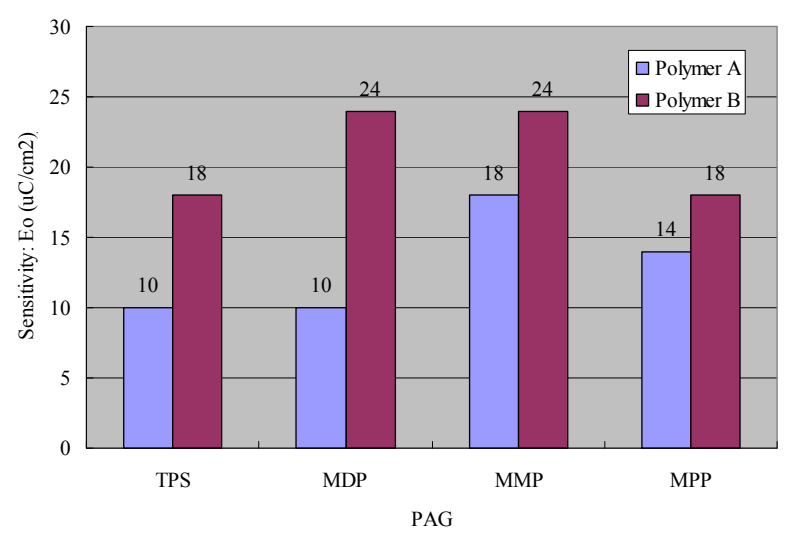

Figure 2. Sensitivity (Eo) comparison

\subsection{Calculation of UV absorption at $13.5 \mathrm{~nm}$}

PAG absorption is a key factor to understand the photolithographic properties under any exposure systems. The PAG absorption at $13.5 \mathrm{~nm}$ was calculated by the method developed by the Center for X-Ray Optics (CXRO), Lawrence Berkeley National Laboratory in the US and used to evaluate the photo-efficiency. [6] The Gas phase transmission of PAG at 295 degree Kelvin under 30 Torr with $1 \mathrm{~cm}$ optical path length was determined by the calculation at CXRO web-site and transformed to absorbance at $13.5 \mathrm{~nm}$. The absorbance is listed in Table 4 as well as the normalized absorbance with that of TPS.

More introduction of alkyl, alkoxy and phenyl group made chromophore absorption higher at 13.5 nm wavelength.

Table 4. PAG absorption at13.5 nm

\begin{tabular}{|c|c|c|}
\hline PAG & $\begin{array}{c}\text { Gas phase } \\
\text { absorbance }\end{array}$ & $\begin{array}{c}\text { Normalized } \\
\text { absorbance }\end{array}$ \\
\hline TPS & 0.718 & 1.00 \\
\hline MDP & 0.888 & 1.24 \\
\hline MMP & 0.861 & 1.20 \\
\hline MPP & 0.988 & 1.38 \\
\hline
\end{tabular}

\subsection{Photolithographic properties in EUV model} formulation

EUV has already achieved the initial requirements for $32 \mathrm{~nm}$ DRAM HP lithography rule and is known as one of the most promising next generation lithography techniques to be realized for $22 \mathrm{~nm}$ patterning technology. [1] In this study, we utilized eMET at Sematech North for $50 \mathrm{~nm} \mathrm{L/S}$ pattering lithography comparing several EUV photoresist model formulations in order to investigate the basic photolithographic properties of PAGs, such as sensitivity, photo-efficiency, exposure latitude and line width roughness (LWR).

\subsubsection{Sensitivity under EUV exposure}

As publically known well, the three severe challenges for EUV lithography are sensitivity, resolution and LWR; the target criterion for each is below $10 \mathrm{~mJ} / \mathrm{cm}^{2}$ in sensitivity, below $40 \mathrm{~nm}$ in HP resolution and below $3 \mathrm{~nm}(3 \sigma)$ in LWR simultaneously. [2]

In the experiments here, $\mathrm{E}_{\text {size }}$, which is a required dose to achieve the desirable $50 \mathrm{~nm} \mathrm{~L} / \mathrm{S}$ pattern lithography, is employed to evaluate the sensitivity for each photoresist. Prior to determination of $E_{\text {size }}$, the critical dimension of the obtained line pattern was determined by CD SEM observation and plotted against the applied dose of energy. From the trend line of the plot, the energy to perform $50 \mathrm{~nm}$ $\mathrm{L} / \mathrm{S}$ patterning was determined and utilized it as $\mathrm{E}_{\text {size }}$ here.

The obtained results are listed in Table 5 and plotted in Figure 3. 
Table 5. Sensitivity $\left(\mathrm{E}_{\text {size }}\right)$ and P-parameter under EUV exposure

\begin{tabular}{|c|c|c|c|c|}
\hline \multirow{2}{*}{ PAG } & \multicolumn{2}{|c|}{$\mathbf{E}_{\text {size }}\left(\mathbf{m J} / \mathbf{c m}^{2}\right)$} & \multicolumn{2}{c|}{$\begin{array}{c}\text { P-parameter } \\
\left(\mathbf{m J} / \mathbf{c m}^{2}\right)\end{array}$} \\
\hline & $\mathrm{A}$ & $\mathrm{B}$ & $\mathrm{A}$ & $\mathrm{B}$ \\
\hline TPS & 11.0 & 12.0 & 11.0 & 12.0 \\
\hline MDP & 9.3 & 9.8 & 11.5 & 12.1 \\
\hline MMP & 10.8 & 9.8 & 13.0 & 11.8 \\
\hline MPP & 11.0 & 11.5 & 15.1 & 15.8 \\
\hline
\end{tabular}

While the significant difference in EB sensitivity was observed between the two formulations, it was interestingly found that there was no clear difference in EUV sensitivity between the two. The fact indicates that acid generation mechanism of EB radiation and EUV exposure are clearly different each other and that the formulations showing inferior sensitivity in EB lithography might perform well in sensitivity under EUV lithography process.

In case of Copolymer A, the value of sensitivity was in the range around $11.0 \mathrm{~mJ} / \mathrm{cm}^{2}$ except MDP. Though the reason why MDP is superior in photospeed is not understood yet, it may be brought by the superior ability to accept the electron transferred from polymer matrix; energy level of LUMO, the energy difference between HOMO and LUMO and distance from hydroxyphenyl moiety are might be key factors in such a case. [9] Further experiments and investigations are necessary to determine the key property in EUV sensitivity results observed here.

Because the reaction of PAGs and thermalized electrons from PHS strongly depends on PAG loading amount and photoresist film thickness in EUV exposure, it is expected optimization of PAG loading amount and photoresist thickness will improve the sensitivity of formulations with Copolymer A. [10-11]

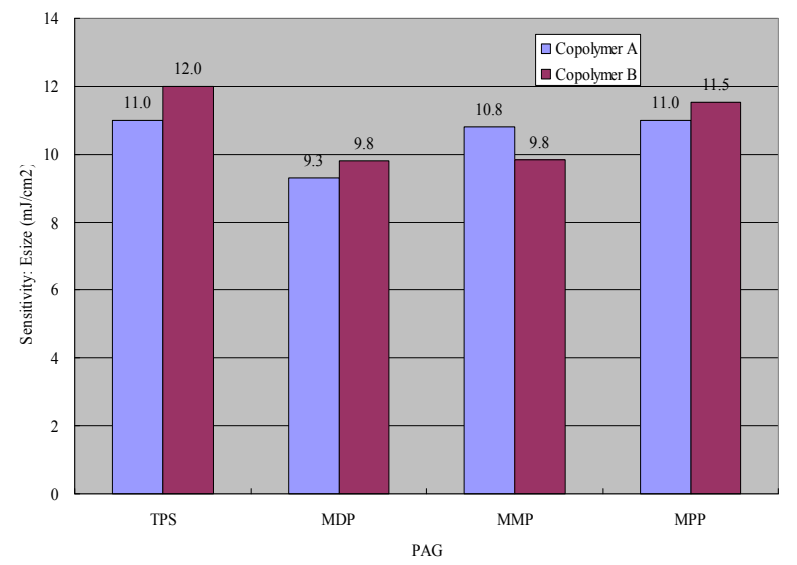

Figure 3. Sensitivity $\left(\mathrm{E}_{\mathrm{size}}\right)$ comparison

\subsubsection{Photo-efficiency under EUV exposure}

It is widely proposed that the electrons generated from PHS polymer matrix of photoresists under EUV radiation are involved in the acid generation reaction from PAG. [12] However less information about the acid generation mechanism in poly(methacrylates) matrix is available. To investigate the possibility of direct excitation of PAG under EUV exposure, values of p-parameter with Copolymer A and Copolymer B were calculated and listed in Table 5. In the case of EUV exposure, $E_{\text {size }}$ was used instead of $E_{0}$ in $\operatorname{ArF}$ and calculated p-parameter normalized by the value of TPS was employed instead of absorbance in acetonitrile.

Normalized p-parameter by the value of TPS is plotted in Figure 4. While the value of TPS, MDP and MMP was varied from 1.00 to 1.18 in case of Copolymer A, the value was interestingly close each other and within the range from 0.98 to 1.01 in case of Copolymer B. Though the reasons of the results observed here are not clear yet, the results may suggest that the acid generation in Copolymer $\mathrm{A}$ is related not directly to PAG absorption but to absorption of matrix, that the generation by the direct excitation of PAG by EUV radiation is dominant in Copolymer B matrix and that the acid generation efficiency from the three PAGs is in the same range. The interpretation supports the proposed acid generation mechanism in PHS. In case of MPP, the p-parameter value in Copolymer $\mathrm{B}$ is significantly inferior to the others. One possible explanation is that the absorption by additional phenyl group on MPP might not contribute to acid generation.

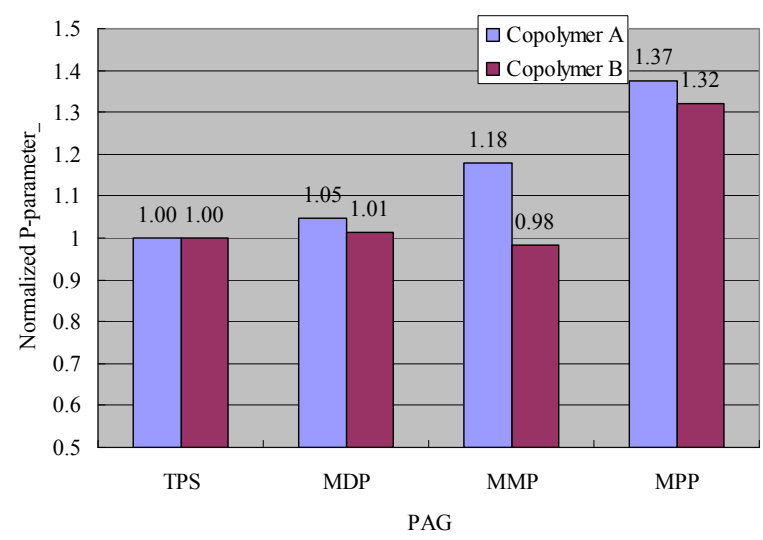

Figure 4. P-parameter (normalized) in copolymers comparison 


\subsubsection{Exposure latitude}

When the lithography process is executed, process latitude of the utilized photoresist is one of the most important factors to be considered. If it is low, high number of defects on lithography process is projected. As a consequence, yield on device manufacturing becomes poor. During the photolithographic studies with eMET, the applied dose of energy was varied. In the trend line of the critical dimension of line vs. the applied doses plot, we employed the slope of the line as a measure to indicate exposure latitude of the photoresists here. If the line width is affected by dose change strongly, the trend line becomes steep and the slope becomes big. The smaller slope value means more tolerant and is more preferable in exposure latitude.

The value of the two types of the model formulations are plotted in Figure 5. It is obvious that exposure latitude of the formulations with Copolymer A is less affected by the sort of PAGs/chromophores and better in performance with any cases except MDP than that with Copolymer B.

One possible explanation for the phenomena observed here is difference in acid generation mechanism as discussed in the sensitivity part; it is known acid generation by the electron transfer from polymer matrix absorbing EUV irradiation is dominant in Copolymer A. [12] In such a case, the acid generation strongly depends on the absorption to PHS matrix. Thus the difference in exposure latitude among PAGs was small. In case of Copolymer B, however, the exposure latitude is affected seriously by the absorption of PAG due to its direct excitation dependency.

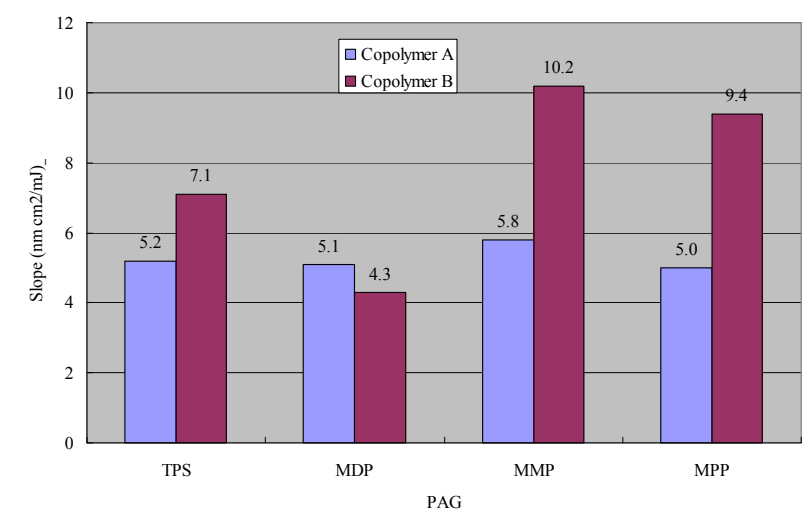

Figure 5. Exposure latitude (slope of critical dimension vs. exposure dose plot) comparison

\subsubsection{Line Width Roughness}

Roughness of the obtained lithography results is becoming a more critical issue with shrinkage of lithography features. In this EUV lithographic study, the resulting LWR in the range from 7.9 to $12.0 \mathrm{~nm}$ was far above the target value of ITRS, below $3 \mathrm{~nm}$. Comparing the LWR results with the determined sensitivity (around $10 \mathrm{~mJ} / \mathrm{cm}^{2}$ ) and achieved resolution $(50 \mathrm{~nm} \mathrm{~L} / \mathrm{S}$ pattern), it is clear that this point is the most serious issue remaining on the model formulations. One way to improve this property is to reduce the acid mobility; the results might be improved by changing acid moiety from nonaflic acid to bulky and less movable one.

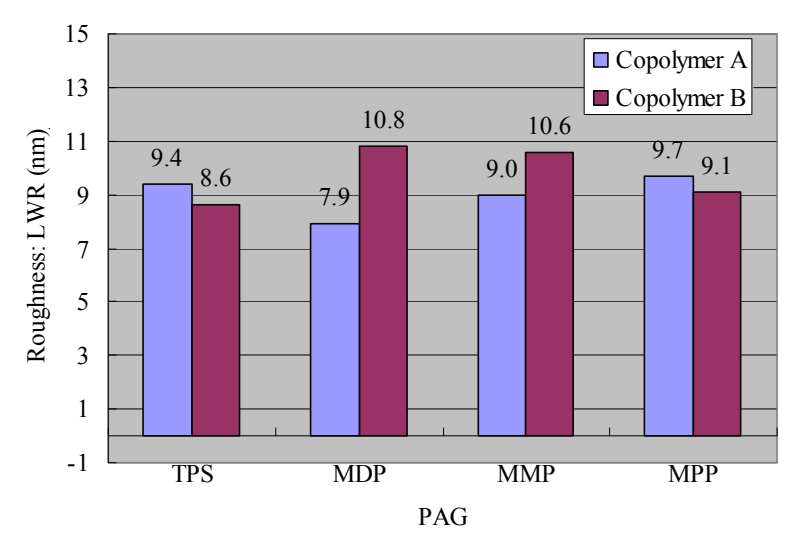

Figure 6. Roughness (LER) Comparison

\section{Conclusion}

In this report, we investigated the fundamental properties of traditional PAG additive approach in EUV lithography with two different polymer platforms, PHS type and poly(methacrylates) type, and four sulfonium nonafluorobutanesulfonate PAGs, TPS, MDP, MMP and MPP, compared with $\mathrm{ArF}$ and $\mathrm{EB}$ exposure techniques to understand the relationship between lithography results and photoresist materials.

While the significant difference in EB sensitivity was observed between the two polymer platforms, it was interestingly found that there was no clear difference in EUV sensitivity between the two. It indicates that acid generation mechanism of EB radiation and EUV exposure are clearly different each other and that the formulations showing inferior sensitivity in EB radiation might perform well in sensitivity under EUV lithography process. Normalized p-parameter values of TPS, MDP and MMP in the Copolymer B were interestingly close each other. It suggests the possibilities that acid the generation by the direct excitation of PAG under EUV radiation is dominant in Copolymer B matrix and that the acid generation efficiency from the three PAGs is in the same range. 


\section{Acknowledgments}

The authors would like to express our sincere thanks to Ms's Rumi Yamashita and Yayoi Kakiuchi for their lab support and to Emil Piscani and Matt Malloy of Sematech North for their experiments on lithography with eMET and to Bill Brunsvold and Nadi Ergenc of Ciba US for their useful discussion and coordination with Sematech North.

\section{References}

1. C. Koh, et al., Proc. SPIE, 7271 (2009) 727124.

2. INTERNATIONAL TECHNOLOGY ROADMAP FOR SEMICONDUCTORS 2008 UPDATE OVERVIEW, $\mathrm{p} 36$.

3. S. Tanaka, et al., Proc. SPIE, 6923 (2008) $69231 \mathrm{~J}$.

4. C-T. Lee, et al., Proc. SPIE, 6519 (2007) 65191E.
5. S. Wurm, et al., Proc. SPIE, 6517 (2007) 651705 .

6. B.L. Henke, E.M. Gullikson, and J.C. Davis, "Xray interactions: photoabsorption, scattering, transmission, and reflection at $\mathrm{E}=50-30000 \mathrm{eV}$, $\mathrm{Z}=1-92$ ", Atomic Data and Nuclear Data Tables 54 (1993) 181-342.

7. G. Pohlers, Y. Suzuki, N. Chan and J. F. Cameron, Proc. SPIE 4690 (2002) 178-190.

8. T. Kozawa, S. Tagawa, et al., J. Photopolym. Sci. Technol., 20 (2007) 577-583.

9. T. Tsuchimura, et al., J. Photopolym. Sci. Technol., 20 (2007) 621-625.

10. R. Hirose, T. Kozawa, et al., Jpn. J. Appl. Phys., 46 (2007) L979-L981.

11. T. Kozawa, S. Tagawa and M. Shell, Jpn. J. Appl. Phys., 46 (2007), L1143-L1145.

12. T. Kozawa, S. Tagawa, et al., J. Vac. Sci. Technol. B, 25 (2007) 2481-2485. 